in the sampling of the commensal microflora and during infection with the pathogenic bacterium Salmonella typhimurium. $C x_{3} c r 1^{\mathrm{GFP} / \mathrm{GFP}}$ mice were more susceptible to infection than heterozygous mice, probably as a result of impaired bacterial sampling and lack of transepithelial dendrites.

This study describes a network of lamina-propria DCs that can directly sample luminal antigens using a mechanism distinct from that involving $\mathrm{M}$ cells. It remains to be determined which antigen-uptake receptors are expressed at the terminal dendrite structures and whether commensal bacteria can be distinguished from pathogens, but the authors suggest that it might be possible to target these structures to engage intestinal DCs, which could be useful for vaccine development.

Elaine Bell

(Q) References and links ORIGinal RESEARCh PAPER Niess, J. H. et al. $\mathrm{CX}_{3} \mathrm{CR} 1$-mediated dendritic cell access to the intestinal lumen and bacterial clearance. Science 307, 254-258 (2005).

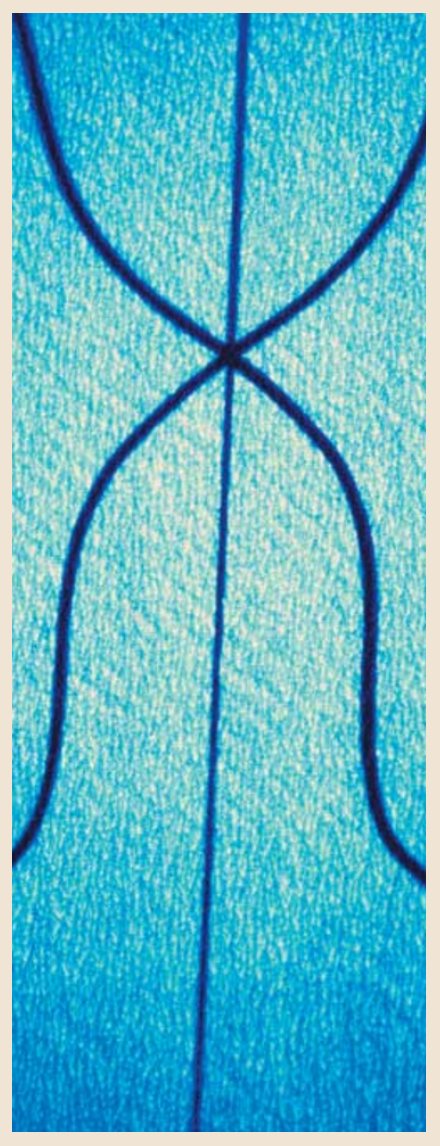

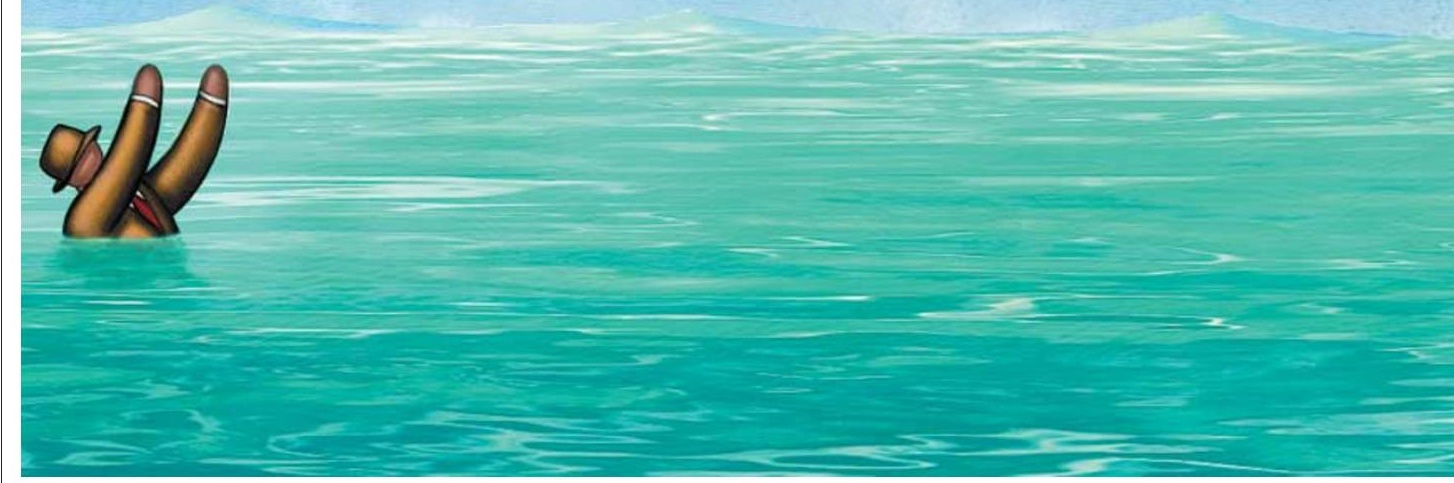

T CELLS

\section{PIM kinases to the rescue}

T-cell survival in the presence of the

immunosuppressive drug rapamycin depends on PIM kinases, as described in a recent report in The Journal of Experimental Medicine. PIM kinases are known to contribute to lymphoid transformation when overexpressed, but until now, their physiological role in the immune response was unclear.

T-cell growth and survival following ligation of cytokine or antigen receptors is mediated, in part, through activation of the effector enzymes AKT and TOR (target of rapamycin) in the phosphatidylinositol 3-kinase pathway. However, alternative pathways of lymphocyte survival are known to exist, because AKT-deficient mice or mice treated with the TOR inhibitor rapamycin mount normal immune responses. In this paper, Craig Thompson and colleagues observed that T cells from mice deficient in PIM1 and PIM2 have increased sensitivity to rapamycin, indicating that PIM kinases might provide this alternative pathway for T-cell survival.

Resting T cells do not express PIM kinases, but PIM kinase expression could be detected in T cells after culturing in the presence of the pro-survival cytokines interleukin-4 (IL-4) and IL-7 or following mitogenic stimulation with $\mathrm{CD} 3$ - and CD28-specific antibodies, with PIM1 expression being detected 3 hours after stimulation and PIM2 expression detected after 12 hours. Culturing of naive T cells with IL-4 and IL-7 also induced phosphorylation and activation of AKT and the TOR subunit p70 S6 kinase, which as expected, could be inhibited by treatment of the $\mathrm{T}$ cells with rapamycin. By contrast, rapamycin treatment did not prevent the induction of PIM protein expression following T-cell stimulation.

In wild-type $\mathrm{T}$ cells or $\mathrm{T}$ cells deficient in PIM1 only, rapamycin treatment had little effect on cytokine-induced survival; however, treatment of PIM2-deficient T cells reduced their ability to survive in response to IL-4 or IL-7, indicating that PIM2 expression is required to compensate for TOR inhibition by rapamycin. The authors suggested that the mechanism of PIM2-dependent survival is likely to be mediated through regulation of the pro-apoptotic BCL-2-related protein $\mathrm{BAD}$ (BCL-2 antagonist of cell death), because phosphorylation of Ser112 of BAD, which contributes to suppression of its pro-apoptotic function, occurred 12 hours after cytokine stimulation, coincident with PIM2 expression.

In addition to having a role in cytokineinduced T-cell survival, the authors show that, in the presence of rapamycin, PIM kinases are required for T-cell blastogenesis, activation and proliferation induced by stimulation with CD3- and CD28-specific antibodies.

Finally, the authors asked whether deficiency in PIM kinases could enhance the immunosuppressive action of rapamycin in vivo. $\mathrm{T}$-cell responses were induced in wild-type or PIM-deficient mice using the superantigen toxicshock syndrome toxin (TSST). Although rapamycin treatment did not affect the activation and clonal expansion of TSST-specific T cells in wild-type mice, PIM-deficient mice had markedly suppressed TSST-induced T-cell responses following rapamycin treatment, consistent with the in vitro observations.

So, although this study shows that PIM kinases can rescue $T$ cells from the immunosuppressive effects of rapamycin both in vitro and in vivo, it does not explain why rapamycin is effective in suppressing transplant rejection in patients who have no known defects in PIM kinases.

LucyBird

(2) References and links

ORIGINAL RESEARCH PAPER Fox, C. J., Hammerman, P. S. \& Thompson, C. B. The Pim kinases control rapamycin-resistant T cell survival and activation. J. Exp. Med. 201, 259-266 (2005). 